\section{Brachial palsy after deep sleep}

A young student presented with left forearm numbness and proximal arm palsy after awaking from benzodiazepine and alcohol consumption-induced deep sleep. The patient had fallen asleep while sitting with the neck leaning on a laptop screen and head tilted left (figure 1). Prior medical history was negative for diabetes mellitus, trauma, neuropathy, chronic alcohol consumption, renal failure or autoimmune disorders. There was no family history of neuropathy. Examination revealed paralysis of the left deltoid, biceps and brachioradialis muscles, mild triceps weakness (4/5 MRC scale), absent biceps tendon reflex and C5-C6 dermatomal hypoesthesia suggesting involvement of the C5-C6-C7 roots or the upper and middle primary trunks of the brachial plexus. Electromyography, performed 3 days after the onset of symptoms, showed no motor unit voluntary recruitment in deltoid, biceps or brachioradialis, and reduced recruitment in triceps. Bilateral upper limb sensory, including lateral antebrachial cutaneous nerve, and motor nerve conduction studies (NCS) were unremarkable. The amplitudes and latencies of compound muscle action potentials recorded from left and right biceps and deltoid muscles were normal and symmetrical even after brachial plexus stimulation at Erb's point.

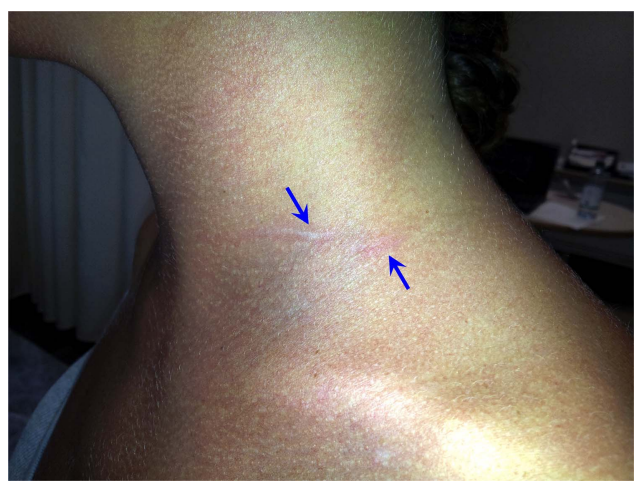

Figure 1 Delayed pressure urticaria (arrow) appeared in left neck area exposed to the maximal pressure.
Nevertheless, a conduction block in nerve segments proximal to Erb's point, not evaluable with conventional NCS, ${ }^{1}$ could not be excluded. Proximal structures can be explored using either magnetic resonance (MR) neurography ${ }^{2}$ or nerve ultrasonography. The latter is sensitive and less expensive and so it could be considered as the preferable investigation. ${ }^{3}$ However, ultrasonography accuracy is highly operator-dependent, and, thus, the choice of imaging technique should also take into consideration local expertise. We decided to perform MR neurography. This revealed hyperintensity and slight swelling of C5-C6-C7 roots and of the middle trunk (figure 2). Somatosensory evoked potentials evoked by upper limb nerve stimulation could also contribute to the functional evaluation of the cervical roots; however, because in our patient MR neurography provided direct evidence of cervical root and brachial plexus involvement, this test was not performed. Genetic analysis for hereditary neuropathy with liability to pressure palsy was negative. Because of the severity of deficits, the patient was treated for 5 days with $1 \mathrm{~g} /$ day intravenous methylprednisolone, even though immunotherapy efficacy is not established for nerve lesions due to mechanical factors. ${ }^{4}$ There was no improvement. After 3 weeks, electromyography showed sporadic fibrillation potentials with minimal recruitment in deltoid, biceps and brachioradialis, and slightly reduced recruitment in triceps. Sensory and motor NCS were unremarkable, suggesting a conduction block with minimal axonal injury in nerve segments proximal to Erb's point. Three months later, the patient showed a pronounced improvement in deltoid, biceps and brachioradialis strength (4/5 MRC) with complete recovery of triceps strength and sensory deficits. Deltoid, biceps and brachioradialis electromyography showed a slightly reduced recruitment. Triceps electromyography was normal.

While sleeping after heavy drinking, arms may be kept in unusual positions, and if they are in contact with a hard surface the pressure can cause peripheral nerve damage. The most common form is the so-called 'Saturday night palsy' in which a compressive lesion of the radial nerve is caused by keeping the arm over the handle of a chair. While a few other cases of pressure-induced brachial plexus lesions have been reported, ${ }^{5}$ this is the first report of a compressive lesion located proximally to the brachial plexus with the involvement of the cervical roots revealed by MR neurography.
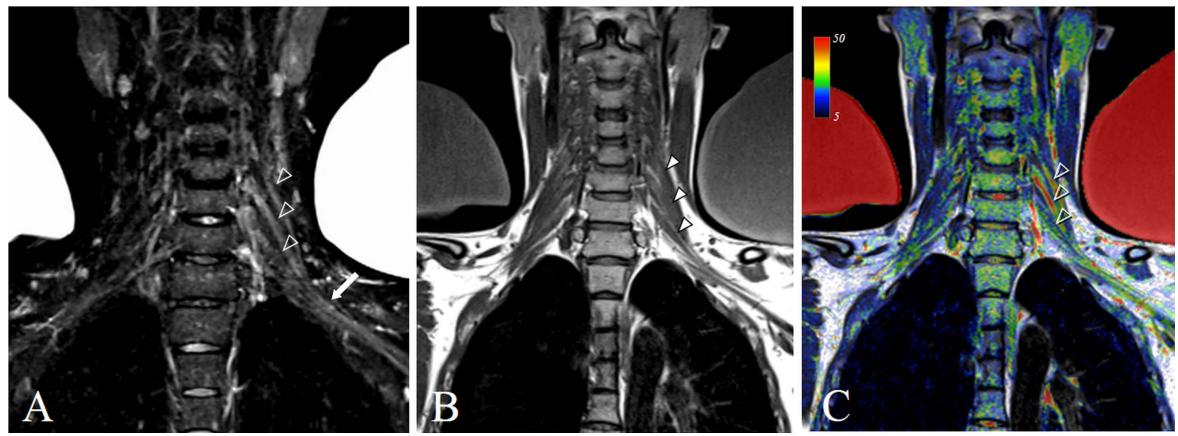

Figure 2 Cervical roots and brachial plexus MRI. Brachial plexus magnetic resonance (MR) neurography was obtained by using maximum intensity-projected short-tau-inversion-recovery (STIR) T2-weighted coronal images. Increased signal and slight swelling of the left brachial plexus C5, C6 and C7 roots (empty arrowheads in A) and middle trunk (arrow in A) indicate oedematous changes. Slight thickening of nerve roots and STIR signal changes are also demonstrated on the corresponding structural T1-weighted (arrowheads in B) and T1/STIR fusion (empty arrowheads in C) images. (MR STIR and T1 images were acquired on a 1.5 T system (Siemens Aera, Erlangen, Germany) with the following parameters: repetition time $=5540 / 700 \mathrm{~ms}$; echo time $=47 / 21 \mathrm{~ms}$; inversion time $=160 /-\mathrm{ms}$; slice thickness $=3.0 \mathrm{~mm}$; gap $=0.3 \mathrm{~mm}$. The percent range of the rendered $\mathrm{T} 2$ intensity values in $\mathrm{C}$ is represented on a colour scale.) 


\section{Vincenzo Di Lazzaro, ${ }^{1,2}$ Federica Giambattistelli, ${ }^{1}$ Emanuele Pravatà, ${ }^{3}$ Giovanni Assenza ${ }^{1}$}

${ }^{1}$ Institute of Neurology Campus Bio-Medico University Via Alvaro del Portillo, Rome, Italy

${ }^{2}$ Fondazione Alberto Sordi, Research Institute for Ageing, Rome, Italy

${ }^{3}$ Neurocenter of Southern Switzerland, via Tesserete 46, Lugano, Switzerland

Correspondence to Professor Vincenzo Di Lazzaro, Institute of Neurology, Campus Bio-Medico University, Via Alvaro del Portillo, 200, Rome 00128, Italy;

v.dilazzaro@unicampus.it

Contributors Drafting/revising the manuscript for content, including medical writing for content, study concept or design, and study supervision or coordination: VDL. Analysis or interpretation of data: VDL, FG, EP and GA. Acquisition of data: VDL, FG, EP and GA.

Patient consent Obtained.

Provenance and peer review Not commissioned; externally peer reviewed.

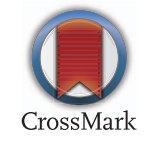

To cite Di Lazzaro V, Giambattistelli F, Pravatà E, et al. J Neurol Neurosurg Psychiatry 2014;85:1409-1410.

Received 26 August 2013

Revised 11 February 2014

Accepted 16 February 2014

Published Online First 12 March 2014

J Neurol Neurosurg Psychiatry 2014;85:1409-1410.

doi:10.1136/jnnp-2013-306637

\section{REFERENCES}

1 Vucic S, Black K, Siao Tick Chong P, et al. Cervical nerve root stimulation. Part II: findings in primary demyelinating neuropathies and motor neuron disease. Clin Neurophysiol 2006;117:398-404.

2 Luigetti M, Pravata E, Colosimo C, et al. MRI neurography findings in patients with idiopathic brachial plexopathy: correlations with clinical-neurophysiological data in eight consecutive cases. Intern Med 2013;52:2031-9.

3 Hobson-Webb LD. Neuromuscular ultrasound in polyneuropathies and motor neuron disease. Muscle Nerve 2013;47:790-804.

4 Staff NP, Engelstad J, Klein CJ, et al. Post-surgical inflammatory neuropathy. Brain 2010;133:2866-80.

5 Fourcade G, Taieb G, Renard D, et al. Brachial plexus sleep palsy. Rev Neurol 2011:167:522-5. 